\title{
Analysis of the fractional order dengue transmission model: a case study in Malaysia
}

\author{
Nur 'Izzati Hamdan ${ }^{1 *}$ and Adem Kilicman ${ }^{1,2}$
}

${ }^{\text {*Correspondence: }}$
izzati.hamdan@gmail.com
${ }^{1}$ Department of Mathematics,
Faculty of Science, Universiti Putra
Malaysia, Selangor, Malaysia
Full list of author information is
available at the end of the article

available at the end of the article

\begin{abstract}
Dengue is one of the important infectious diseases in the world. In Malaysia, dengue occurs nationally and has been endemic for more than a decade. Hence, the modeling of dengue transmission is of great importance to help us understand the dynamical behavior of the disease. In this paper, we developed a compartmental model of the dengue transmission using the fractional order differential equation. It consists of six compartments representing the human and mosquito dynamics. The disease-free and the positive endemic equilibrium point are obtained. The stability analysis of the equilibria is presented. A sensitivity analysis of the model is performed to determine the relative importance of the model parameters to the transmission. Numerical simulations are given for different parameter settings. A case study, using the outbreak dengue data in the state of Selangor, Malaysia, in 2012, is presented.
\end{abstract}

Keywords: Fractional system; Dengue; Stability analysis; Reproduction number; Sensitivity analysis

\section{Introduction}

Dengue fever, commonly known as dengue, is a painful, debilitating mosquito-borne tropical disease caused by the dengue virus. It is a viral disease transmitted by the bite of an Aedes mosquito infected with any of the four serotypes, denoted by DEN-I, DEN-II, DEN-III, and DEN-IV, respectively. In recent decades, the spread of the dengue virus has increased rapidly and according to the World Health Organization (WHO) there are millions of dengue cases reported every year worldwide [1]. A human that gets infected by any of these dengue serotype produces permanent immunity to it, but only a temporary cross-immunity to the other serotypes [2].

In Malaysia, dengue occurs nationally with increased risk in urban and peri-urban areas. The number of dengue fever cases reported in our nation continues to increase since 2010 [3]. There is no specific vaccine available for dengue in Malaysia. Preventing and controlling the dengue virus depends solely on the control of the mosquito vector or interruption of human-vector contact. Hence, a reliable mathematical model is essential to give a deeper understanding of the mechanism of dengue transmission and on how to control the spread of the disease.

The well-known susceptible-infected-recovered (SIR) epidemic model introduced by Kermack and McKendrick in 1927 [4] has been used by many researchers to study the dynamics of the infectious disease. Later, Bailey [5] developed a simple vector-host dengue

(c) The Author(s) 2019. This article is distributed under the terms of the Creative Commons Attribution 4.0 International License (http://creativecommons.org/licenses/by/4.0/), which permits unrestricted use, distribution, and reproduction in any medium, provided you give appropriate credit to the original author(s) and the source, provide a link to the Creative Commons license, and indicate if changes were made. 
transmission model for a single serotype based on the SIR epidemic model. The development of the dengue transmission model has become attention ever since, parallel to the increase in the number of dengue cases reported all around the world. Most of the model is based on the ordinary differential equation [6-10].

In the last few years, fractional order calculus is found to be more interesting in modeling a real problem in comparison to a classical integer order as it provides a tool for the description of memory effects and genetic properties of various materials [11-14]. In this paper, the proposed dengue epidemic model is derived using the generalized fractional order derivative.

Pooseh et al. [15] were the first that fractionalized the dengue ODE model using the Riemann-Liouville definition. Later, Diethelm [16] proposed a more sophisticated way of fractional dengue model by using the Caputo definition. Diethelm solved the problem of mismatched dimension in the earliest model by Pooseh et al. Sardar et al. in $[17,18]$ showed the significance of order incorporated with memory in the dengue transmission. In this paper, we will not only be considered the adult stage of mosquito, but also the dynamic of the aquatic stage of mosquito. We will be considered the work of Diethelm [16] in fractionalize the ODE system, but with the assumption that any parameter related to the birth and death in both human and mosquito population is assumed to be memory independent.

The rest of the paper is organized as follows. In Sect. 2, the formulation of the model is presented. The stability analysis of the equilibrium points is given in Sect. 3. In Sect. 4, the sensitivity analysis is performed. Section 5 consists of the numerical experiments done to verify the theoretical analysis shown in Sects. 3 and 4. Lastly, the conclusion is in Sect. 6.

\section{Mathematical model}

For many years, fractional operators have been interpreted in several ways that fit the concept of their integrals or derivatives. For instance, the most common definitions of the fractional derivative are the Riemann-Liouville derivative, the Caputo derivative, and the Grünwald-Letnikov derivative. In this paper, the Caputo derivative is used, since the classical initial conditions can be used without encountering any problem during obtaining the solutions.

The definition of the Caputo derivative is the following equation:

$$
D_{C}^{\alpha} f(x)=\frac{1}{\Gamma(n-\alpha)} \int_{a}^{x} \frac{d^{n} f}{d \varepsilon^{n}}(\varepsilon)(x-\varepsilon)^{n-\alpha-1} d \varepsilon,
$$

where $\alpha$ is the order of the derivative with $n-1<\alpha<n$ and $n=[\alpha]+1 . \Gamma(n-\alpha)$ is the Euler gamma function defined by the so-called Euler integral of the second kind [19]:

$$
\Gamma(n-\alpha)=\int_{0}^{\infty} t^{n-\alpha-1} e^{-t} d t
$$

where $t^{n-\alpha-1}=e^{(n-\alpha-1) \log (t)}$.

In the formulation of the model, we assume that the total human and vector population to be constant. We also assume that the infection is produced by only one serotype of dengue virus. The dynamics of the female Aedes mosquito are included aquatic stage $\left(A_{m}\right)$ and adult stage. The adult female mosquito $(M)$ is divided into two compartments that are 
susceptible $M_{s}$ and infected $M_{i}$. The total human population, $N_{h}(t)=H$ is partitioned into three compartments: susceptible, $H_{s}$, infected, $H_{i}$, and recovered, $H_{r}$ individuals.

The system of fractional order nonlinear differential equation for the proposed hostvector model is as follows:

$$
\begin{aligned}
& D^{\alpha} A_{m}=q \phi\left(1-A_{m} / C\right) M-\left(\sigma_{A}+\mu_{A}\right) A_{m}, \\
& D^{\alpha} M_{s}=\sigma_{A} A_{m}-\frac{b^{\alpha} \beta_{m}}{H} M_{s} H_{i}-\mu_{m} M_{s}, \\
& D^{\alpha} M_{i}=\frac{b^{\alpha} \beta_{m}}{H} M_{s} H_{i}-\mu_{m} M_{i}, \\
& D^{\alpha} H_{s}=\mu_{h}\left(H-H_{s}\right)-\frac{b^{\alpha} \beta_{h}}{H} H_{s} M_{i}, \\
& D^{\alpha} H_{i}=\frac{b^{\alpha} \beta_{h}}{H} H_{s} M_{i}-\left(\gamma_{h}+\mu_{h}+\rho_{h}\right) H_{i}, \\
& D^{\alpha} H_{r}=\gamma_{h} H_{i}-\mu_{h} H_{r} .
\end{aligned}
$$

The parameter $H$ is the total human population, $q$ is the proportion of eggs that results in female mosquito, $\sigma_{A}$ is the transition rate from aquatic stage to adult stage, $\mu_{A}$ is the per capita mortality rate of aquatic phase, $1 / \mu_{m}$ is the average lifespan of adult mosquitoes, $1 / \mu_{h}$ is the average lifespan of human, $b$ is the biting rate, $\beta_{m}$ is the transmission probability from human to vector, $\beta_{h}$ is the transmission probability from vector to human, $\gamma_{h}$ is the recovery rate in the host population, $C$ is the aquatic carrying capacity, $m$ is the number of female mosquitoes per human, $k$ is the number of larvae per human, $\phi$ is the oviposition rate and $\rho_{h}$ is the per capita disease-induced death rate for humans. All parameters in (3) are assumed to be non-negative as the system monitors the dynamics of the human population. The possible feasible ranges of each parameter of model (3) are given in Table 1.

Since $N_{h}=H_{s}+H_{i}+H_{r}$, we have $H_{r}=N_{h}-H_{s}-H_{i}$. Therefore, we can simplify system (3) to the following system:

$$
\begin{aligned}
& D^{\alpha} A_{m}=q \phi\left(1-A_{m} / C\right) M-\left(\sigma_{A}+\mu_{A}\right) A_{m}, \\
& D^{\alpha} M_{s}=\sigma_{A} A_{m}-\frac{b^{\alpha} \beta_{m}}{H} M_{s} H_{i}-\mu_{m} M_{s}, \\
& D^{\alpha} M_{i}=\frac{b^{\alpha} \beta_{m}}{H} M_{s} H_{i}-\mu_{m} M_{i}, \\
& D^{\alpha} H_{s}=\mu_{h}\left(H-H_{s}\right)-\frac{b^{\alpha} \beta_{h}}{H} H_{s} M_{i}, \\
& D^{\alpha} H_{i}=\frac{b^{\alpha} \beta_{h}}{H} H_{s} M_{i}-\left(\gamma_{h}+\mu_{h}+\rho_{h}\right) H_{i} .
\end{aligned}
$$

\section{Existence and stability of equilibrium points}

Suppose that the set $\Omega=\left\{\left(A_{m}, M_{s}, M_{i}, H_{s}, H_{i}, H_{s}\right) \in \mathbb{R}_{+}^{5}: H_{s}+H_{i} \leq K ; 0 \leq M_{s}+M_{i} \leq\right.$ $Q_{1}$, and $Q_{1} \geq \frac{\sigma_{A} A}{\mu_{m}} ; 0 \leq A_{m} \leq Q_{2}$, and $\left.Q_{2} \geq q \phi M\right\}$ is the region of biological interest, that is, positively invariant to the system (4). The proof is similar to the proof in [20].

From system (4), for all parameter values, it is straightforward to obtain a disease-free equilibrium $E_{0}=(0,0,0, H, 0)$. Using Maple software, we obtained one more disease-free 
Table 1 Description of the dengue model (3) parameters and their possible feasible ranges

\begin{tabular}{llll}
\hline Parameter & Biological meaning & Range of values & References \\
\hline$q$ & Proportion of eggs & $0-1$ & {$[8]$} \\
$\phi$ & Oviposition rate & $0-11.2$ per day & {$[8]$} \\
$\sigma_{A}$ & Transition rate from aquatic to adult & $0-0.19$ per day & {$[8]$} \\
$\mu_{A}$ & Average aquatic mortality rate & $0.01-0.47$ per day & {$[8]$} \\
$1 / \mu_{m}$ & Average lifespan of adult mosquito & $11-56$ days & {$[21]$} \\
$1 / \mu_{h}$ & Average lifespan of human & $73-75$ years & {$[22]$} \\
$b$ & The biting rate & $0-1$ per day & {$[23]$} \\
$\beta_{m}$ & Transmission probability from human to vector & 0.375 & {$[6]$} \\
$\beta_{h}$ & Transmission probability from vector to human & 0.375 & {$[6]$} \\
$\gamma_{h}$ & Recovery rate in the host population & 0.328833 per day & {$[21]$} \\
$\rho_{h}$ & Disease-induced death rate for humans & $10^{-3}$ & {$[24]$} \\
\hline
\end{tabular}

equilibrium,

$$
E_{1}=\left(\overline{A_{m}}, \bar{M}_{s}, 0, H, 0\right)
$$

where $\overline{A_{m}}$ and $\bar{M}_{s}$ are given by

$$
\overline{A_{m}}=C\left(1-\frac{1}{R_{m}}\right) \text { and } \bar{M}_{s}=\frac{\sigma_{A} \overline{A_{m}}}{\mu_{m}} .
$$

Thus we obtained the following:

$$
\begin{aligned}
& E_{1}=\left(\frac{C\left(q \phi \sigma_{A}-\mu_{A} \mu_{m}-\mu_{m} \sigma_{A}\right)}{q \phi \sigma_{A}}, \frac{C\left(q \phi \sigma_{A}-\mu_{A} \mu_{m}-\mu_{m} \sigma_{A}\right)}{q \phi \mu_{m}}, 0, H, 0\right), \\
& E_{1}=\left(C\left(1-\frac{1}{R_{m}}\right), \frac{C \sigma_{A}}{\mu_{m}}\left(\frac{R_{m}-1}{R_{m}}\right), 0, H, 0\right),
\end{aligned}
$$

where $R_{m}=\frac{q \phi \sigma_{A}}{\mu_{m}\left(\sigma_{A}+\mu_{A}\right)} . R_{m}$ denotes the 'basic offspring' of the mosquito population. This results in the following proposition.

Proposition 1 Consider $R_{m}$ to be the basic offspring of the mosquito population

$$
R_{m}=\frac{q \phi \sigma_{A}}{\mu_{m}\left(\sigma_{A}+\mu_{A}\right)}
$$

The system of equations (4) has at most two disease-free equilibrium points:

- if $R_{m} \leq 0$, there is a disease-free equilibrium (DFE), known as trivial equilibrium, $E_{0}=(0,0,0, H, 0)$;

- if $R_{m}>0$, there is a biologically realistic disease-free equilibrium (BRDFE), $E_{1}=\left(\bar{A}_{m}, \bar{M}_{s}, 0, H, 0\right)$.

or an endemic equilibrium $E_{2}=\left(A_{m}^{*}, M_{s}^{*}, M_{i}^{*}, H_{s}^{*}, H_{i}^{*}\right)$.

In modeling the infectious disease, the basic reproduction number $R_{0}$ is important. This threshold quantity value represents the expected number secondary cases produced in a completely susceptible population, by a typical infected individual during its entire period of infectious [25]. In this paper we use the next-generation matrix approach to obtaining the $R_{0}$. By following [26], we are thus lead to the following proposition. 
Proposition 2 If $R_{m}>0$, then the basic reproduction number associated to the system (4) is $R_{0}^{2}=\frac{b^{2 \alpha} \beta_{m} \beta_{h}}{\left(\gamma_{h}+\mu_{h}+\rho_{h}\right) \mu_{m}} \frac{\bar{M}_{s}}{H}$. The BRDFE is locally asymptotically stable if $R_{0}<1$ and unstable otherwise.

The local stability of the BRDFE $E_{1}$ is governed by the eigenvalues of the Jacobian matrix of system (4) about $E_{1}$, which can be computed as

$$
J\left(E_{1}\right)=\left(\begin{array}{ccccc}
-R_{m}\left(\sigma_{A}+\mu_{A}\right) & 0 & 0 & 0 & 0 \\
\sigma_{A} & -\mu_{m} & 0 & 0 & \frac{b^{\alpha} \beta_{m}}{H} \bar{M}_{s} \\
0 & 0 & -\mu_{m} & 0 & \frac{b^{\alpha} \beta_{m}}{H} \bar{M}_{s} \\
0 & 0 & -b^{\alpha} \beta_{h} & -\mu_{h} & 0 \\
0 & 0 & b^{\alpha} \beta_{h} & 0 & -\left(\gamma_{h}+\mu_{h}+\rho_{h}\right)
\end{array}\right) .
$$

The calculated eigenvalues are $\lambda_{1}=-R_{m}\left(\sigma_{A}+\mu_{A}\right), \lambda_{2}=-\mu_{m}, \lambda_{3}=-\mu_{h}$. the others are the roots of the second order polynomial

$$
\lambda^{2}+\left(\gamma_{h}+\mu_{h}+\rho_{h}+\mu_{m}\right) \lambda+\mu_{m}\left(\gamma_{h}+\mu_{h}+\rho_{h}\right)\left(1-R_{0}^{2}\right)
$$

By the Routh-Hurwitz stability criterion, $E_{1}$ is locally asymptotically stable if and only if $R_{0}<1$, otherwise the BRDFE is unstable.

We assumed that $E_{2}=\left(A_{m}^{*}, M_{s}^{*}, M_{i}^{*}, H_{s}^{*}, H_{i}^{*}\right)$ is any arbitrary positive endemic equilibrium of the reduced system (4). It is convenient to express the variables of the model at the steady state in terms of the force of infection denoted by $\lambda_{m}^{*}$ and $\lambda_{h}^{*}$ :

$$
\begin{aligned}
& \lambda_{m}^{*}=\frac{b^{\alpha} \beta_{m} H_{i}}{H}, \\
& \lambda_{h}^{*}=\frac{b^{\alpha} \beta_{h} M_{i}}{H} .
\end{aligned}
$$

Thus, system (3) can be written as follows:

$$
\begin{aligned}
& D^{\alpha} A_{m}=q \phi\left(1-A_{m} / C\right) M-\left(\sigma_{A}+\mu_{A}\right) A_{m}, \\
& D^{\alpha} M_{s}=\sigma_{A} A_{m}-\lambda_{m}^{*} M_{s}-\mu_{m} M_{s} \\
& D^{\alpha} M_{i}=\lambda_{m}^{*} M_{s}-\mu_{m} M_{i} \\
& D^{\alpha} H_{s}=\mu_{h}\left(H-H_{s}\right)-\lambda_{h}^{*} H_{s} \\
& D^{\alpha} H_{i}=\lambda_{h}^{*} H_{s}-\left(\gamma_{h}+\mu_{h}+\rho_{h}\right) H_{i} .
\end{aligned}
$$

Now we solve Eq. (4) at the steady state $E_{2}$, we have the following endemic equilibrium in terms of $\lambda_{m}^{*}$ and $\lambda_{h}^{*}$ :

$$
\begin{aligned}
& A_{m}^{*}=\overline{A_{m}}=C\left(1-\frac{1}{R_{m}}\right), \\
& M_{s}^{*}=\frac{\sigma_{A} C\left(1-\frac{1}{R_{m}}\right)}{\lambda_{m}^{*}+\mu_{m}}, \\
& M_{i}^{*}=\frac{\lambda_{m}^{*}}{\mu_{m}} \frac{\sigma_{A} C\left(1-\frac{1}{R_{m}}\right)}{\lambda_{m}^{*}+\mu_{m}},
\end{aligned}
$$




$$
\begin{aligned}
& H_{s}^{*}=\frac{\mu_{h} H}{\mu_{h}+\lambda_{h}^{*}} \\
& H_{i}^{*}=\frac{\lambda_{h}^{*} \mu_{h} H}{\left(\gamma_{h}+\mu_{h}+\rho_{h}\right)\left(\mu_{h}+\lambda_{h}^{*}\right)} .
\end{aligned}
$$

Substitute (7) in (5), and simplify:

$$
\begin{aligned}
& \lambda_{m}^{*}=\frac{b^{\alpha} \beta_{m} \mu_{h} \lambda_{h}^{*}}{\left(\gamma_{h}+\mu_{h}+\rho_{h}\right)\left(\mu_{h}+\lambda_{h}^{*}\right)}, \\
& \lambda_{h}^{*}=\frac{b^{\alpha} \beta_{h} \lambda_{m}^{*} \sigma_{A} C\left(1-\frac{1}{R_{m}}\right)}{H \mu_{m}\left(\lambda_{m}^{*}+\mu_{m}\right)} .
\end{aligned}
$$

Replacing (8) and (7) in (5) and using the expression of $R_{0}$, we will have $\lambda_{m}^{*}$ and $\lambda_{h}^{*}$ in terms of $R_{0}$ as follows:

$$
\begin{aligned}
& \lambda_{m}^{*}=\frac{\mu_{m}\left(R_{0}^{2}-1\right)}{1+\mu_{m}\left(\gamma_{h}+\mu_{h}+\rho_{h}\right) R_{0}^{2}}, \\
& \lambda_{h}^{*}=\frac{\mu_{m} \mu_{h}\left(\gamma_{h}+\mu_{h}+\rho_{h}\right)\left(R_{0}^{2}-1\right)}{b^{\alpha} \beta_{m} \mu_{h}+\mu_{m}\left(\gamma_{h}+\mu_{h}+\rho_{h}\right)} .
\end{aligned}
$$

Using the relation in (9), we express $\left(A_{m}^{*}, M_{s}^{*}, M_{i}^{*}, H_{s}^{*}, H_{i}^{*}\right)$ in (7) in terms of $R_{0}$ :

$$
\begin{aligned}
& A_{m}^{*}=\overline{A_{m}}=C\left(1-\frac{1}{R_{m}}\right) \\
& M_{s}^{*}=\frac{\sigma_{A} C\left(1-\frac{1}{R_{m}}\right)\left(1+\mu_{m}\left(\gamma_{h}+\mu_{h}+\rho_{h}\right) R_{0}^{2}\right)}{\mu_{m} K_{1} R_{0}^{2}}, \\
& M_{i}^{*}=\frac{\sigma_{A} C\left(1-\frac{1}{R_{m}}\right)\left(R_{0}^{2}-1\right)}{\mu_{m} K_{1} R_{0}^{2}}, \\
& H_{s}^{*}=\frac{H K_{2}}{K_{2}+\mu_{m}\left(\gamma_{h}+\mu_{h}+\rho_{h}\right)\left(R_{0}^{2}-1\right)}, \\
& H_{i}^{*}=\frac{H \mu_{m} \mu_{h}\left(R_{0}^{2}-1\right)}{K_{2}+\mu_{m}\left(\gamma_{h}+\mu_{h}+\rho_{h}\right)\left(R_{0}^{2}-1\right)},
\end{aligned}
$$

where $K_{1}=1+\mu_{m}\left(\gamma_{h}+\mu_{h}+\rho_{h}\right)$ and $K_{2}=b^{\alpha} \beta_{m} \mu_{h}+\mu_{m}\left(\gamma_{h}+\mu_{h}+\rho_{h}\right)$.

Therefore, $R_{0}>1$, it implies $R_{0}^{2}>1$, and, as a result, from (10) there exists a unique positive endemic equilibrium $E_{2}=\left(A_{m}^{*}, M_{s}^{*}, M_{i}^{*}, H_{s}^{*}, H_{i}^{*}\right)$. Thus, we have the following result.

Theorem 1 The reduced system (4) corresponding to model (3) has a unique positive endemic equilibrium $E_{2}=\left(A_{m}^{*}, M_{s}^{*}, M_{i}^{*}, H_{s}^{*}, H_{i}^{*}\right)$ if $R_{0}>1$.

\section{Sensitivity analysis}

Sensitivity analysis helps us to identify the parameters that have a big impact on the disease transmission. Such information is important not only for experimental design but also for data assimilation and reduction to complex nonlinear models [27]. Normally, in the epidemiological model, the analysis is used to discover parameters that have greatest influence on the basic reproduction number $R_{0}$ and should be targeted by the control strategies. 
The sensitivity indices of the $R_{0}$ are determined to allow us to measure which parameter has the greatest influence on the changes of $R_{0}$ and, hence, the greatest effect in determining whether the disease can be eliminated in the population. The normalized forward sensitivity index of a variable $\left(R_{0}\right)$ with respect to a parameter is the ratio of the relative change in the variable $\left(R_{0}\right)$ to the relative change in the parameter. When the variable is a differentiable function of the parameter, the sensitivity index can be defined using the partial derivatives [27].

Definition 1 (cf. [28]) The normalized forward sensitivity index of $R_{0}$, which depends differentiably on a parameter $p$, is defined by

$$
\Upsilon_{p}^{R_{0}}=\frac{\partial R_{0}}{\partial p} \times \frac{p}{R_{0}} .
$$

Recall that the value of $R_{0}$ for the system (4) is given by

$$
R_{0}^{2}=\frac{b^{2 \alpha} \beta_{m} \beta_{h}}{\left(\gamma_{h}+\mu_{h}+\rho_{h}\right) \mu_{m}} \frac{\bar{M}_{s}}{H} .
$$

The index indicates how sensitive the variable $R_{0}$ is to the change of parameter $p$. The positive index clearly reveals that an increase in the parameter value will result in an increase of the $R_{0}$ value and vice versa. The index values for the parameters in (4) are presented in Tables 2 and 3. The graphical presentation of the sensitivity index for each parameter with the order of the derivative is $\alpha=0.9$ can be found in Fig. 1.

Table 2 Sensitivity indices of $R_{0}$ evaluated at the baseline parameter values for $\alpha=0.9$

\begin{tabular}{ll}
\hline Parameter & Sensitivity index \\
\hline$q$ & -0.0097 \\
$\phi$ & -0.0097 \\
$\sigma_{A}$ & +0.5073 \\
$\mu_{A}$ & +0.0073 \\
$\mu_{m}$ & -1.0097 \\
$\mu_{h}$ & $-5.5798 \times 10^{-5}$ \\
$b$ & +0.90 \\
$\beta_{m}$ & +0.50 \\
$\beta_{h}$ & +0.50 \\
$\gamma_{h}$ & -0.5092 \\
$\rho_{h}$ & -0.0015 \\
\hline
\end{tabular}

Table 3 Sensitivity indices of $R_{0}$ evaluated at the baseline parameter values for $\alpha=0.6$

\begin{tabular}{ll}
\hline Parameter & Sensitivity index \\
\hline$q$ & -0.0097 \\
$\phi$ & -0.0097 \\
$\sigma_{A}$ & +0.5073 \\
$\mu_{A}$ & +0.0073 \\
$\mu_{m}$ & -1.0097 \\
$\mu_{h}$ & $-5.5798 \times 10^{-5}$ \\
$b$ & +0.60 \\
$\beta_{m}$ & +0.50 \\
$\beta_{h}$ & +0.50 \\
$\gamma_{h}$ & -0.5092 \\
$\rho_{h}$ & -0.0015 \\
\hline
\end{tabular}




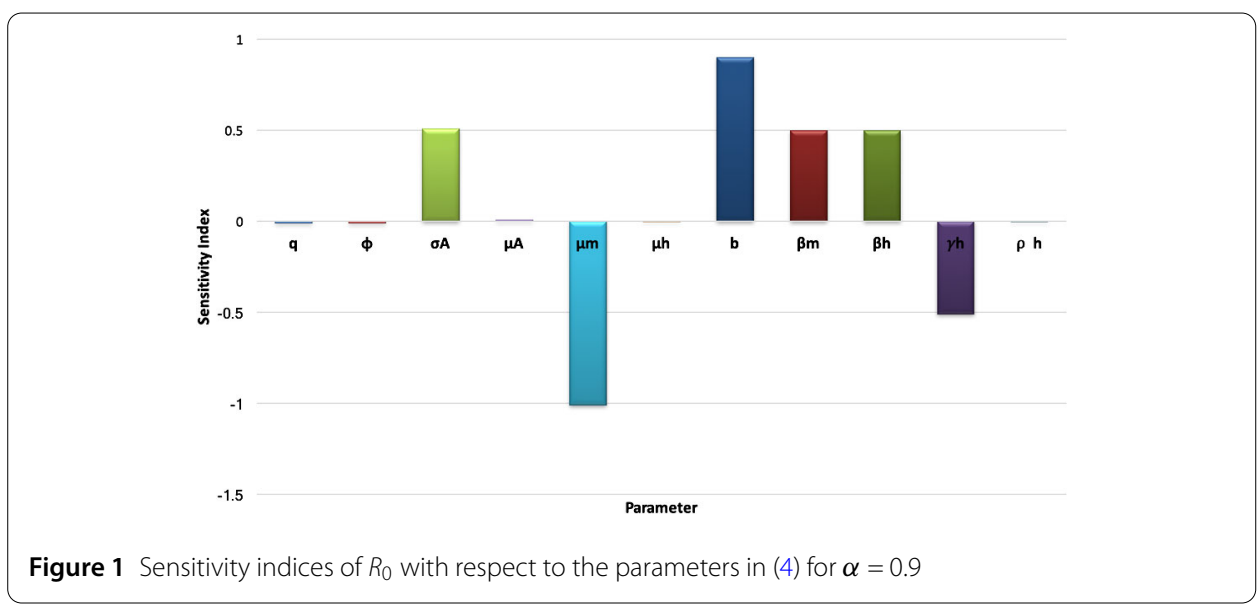

Figure 2 Solutions of the model (3) and the real data coming from the dengue cases recorded in Selangor, Malaysia, in 2012

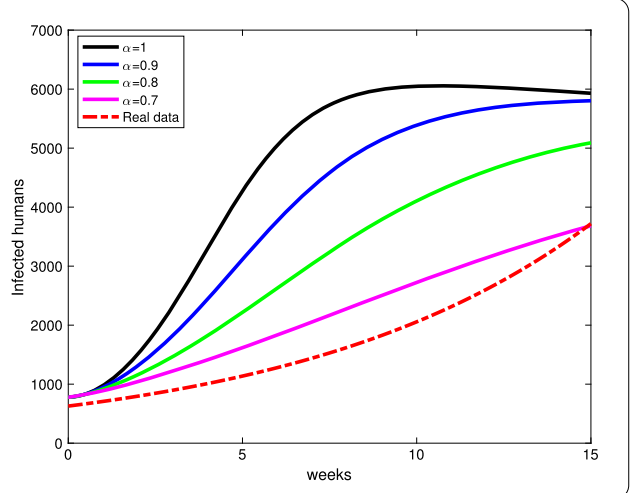

\section{Numerical simulations and discussion}

The simulations were carried out using the following initial conditions based on the dengue cases reported in Selangor for the year 2012 [29]:

$$
\begin{aligned}
& H_{s 0}=N_{h}-H_{i 0}, \quad H_{i 0}=7799, \\
& A_{m 0}=k N_{h}, \quad M_{s 0}=m N_{h},
\end{aligned}
$$

where $N_{h}=5,650,800, k=3, m=4$. The final time $t_{\text {end }}=365$ days. The computation is done in Matlab using the code fde12 [30].

The selection of order $\alpha$ is similar to the approach taken in the previous work [20]. We solved the system for a various choice of $\alpha$ values. Specifically, we have used the formula of $\alpha \in\{k / 100: k=1,2, \ldots, 100\}$. From that, we found that a reasonable range of $\alpha$ is between 0.7 and 1 . Note that $\alpha=1$ gives the same solution as the integer order system.

In Fig. 2, we present the solutions of our model during a shorter interval of time in weeks and also the real data fitted curve for the same time interval based on the data collected. From Fig. 2, we can observe that the fractional model provides a better approximation towards the real data compared to the classical integer order solution $(\alpha=1)$.

Figures 3 and 4 show the solutions to (4) with the initial conditions (13). It can be seen in Fig. 3 that the solutions approach the stable disease-free equilibrium when $R_{0}$ is less than 1 . Notice that the speed of convergence is decreased, once the order $\alpha$ is reduced. The same behavior is observed in Fig. 4 for the speed of convergence. Here, $R_{0}>1$, and 


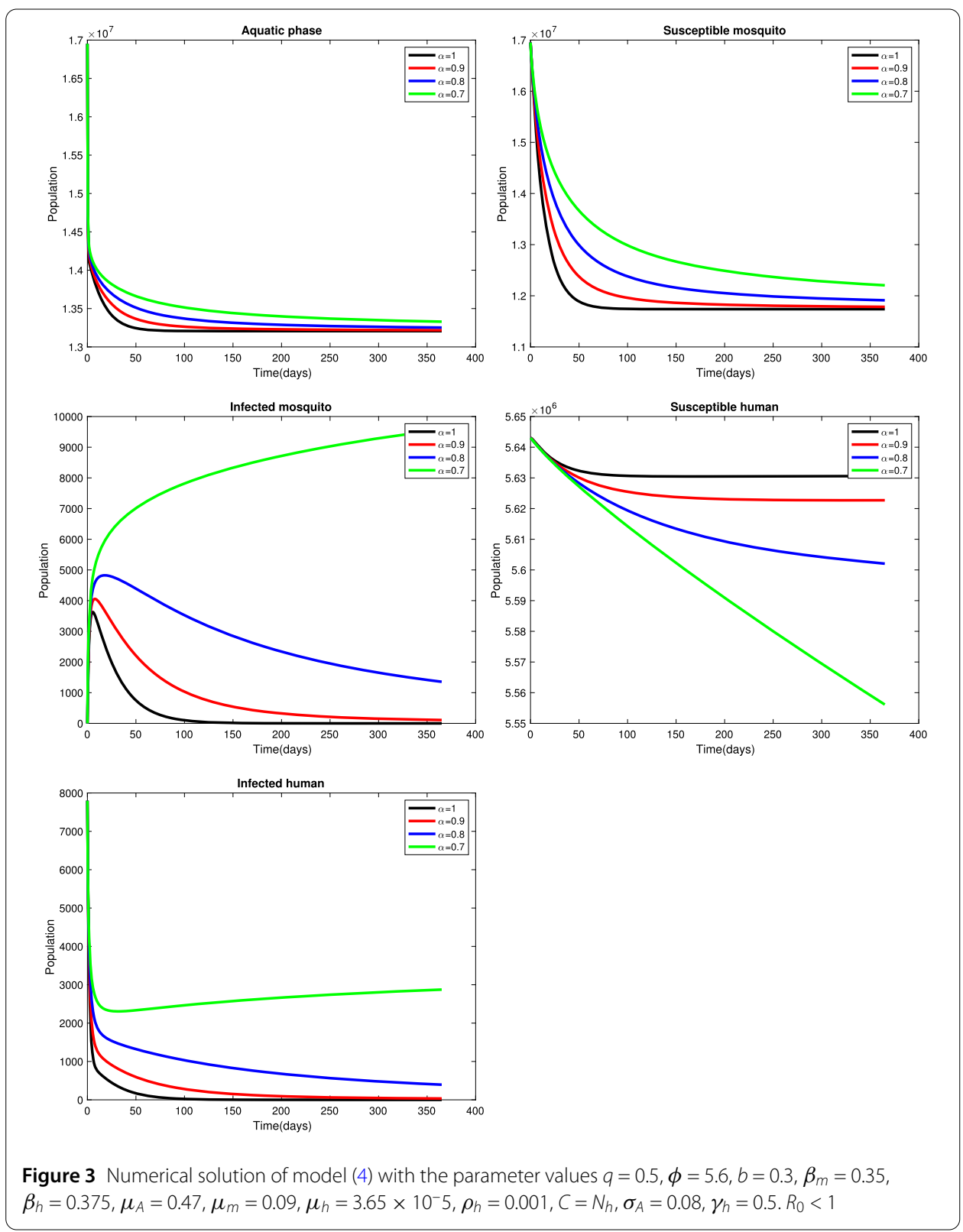

we can verify that the system approaches the stable endemic equilibrium over time. This indicates that the disease is persistent in the community and is endemic over a long period of time.

Figure 5 shows graphs of the infected human population through parameter variation. The obtained figure reinforces the sensitivity analysis made in Sect. 4. We choose to plot graphs of the different parameter that gives high influence on $R_{0}$, in other words, having a big sensitivity index. From Table 2 , the most positive parameter is the mosquito biting rate, $b$, where the index is 0.9. Then we have $\sigma_{A}$ and $\beta_{m}, \beta_{h}$.

Notice that, in Table 3, the index of the parameter $b$ is decreased as we reduced the order $\alpha$. This explained the relationship of $R_{0}$ with the $\alpha$. The threshold quantity $R_{0}$ defined here is a memory-dependent threshold quantity as $R_{0} \propto b^{\alpha}$. As the memory of the biting rate of the mosquito increased ( $\alpha \rightarrow 0)$, the basic reproduction number increased, thus, we 


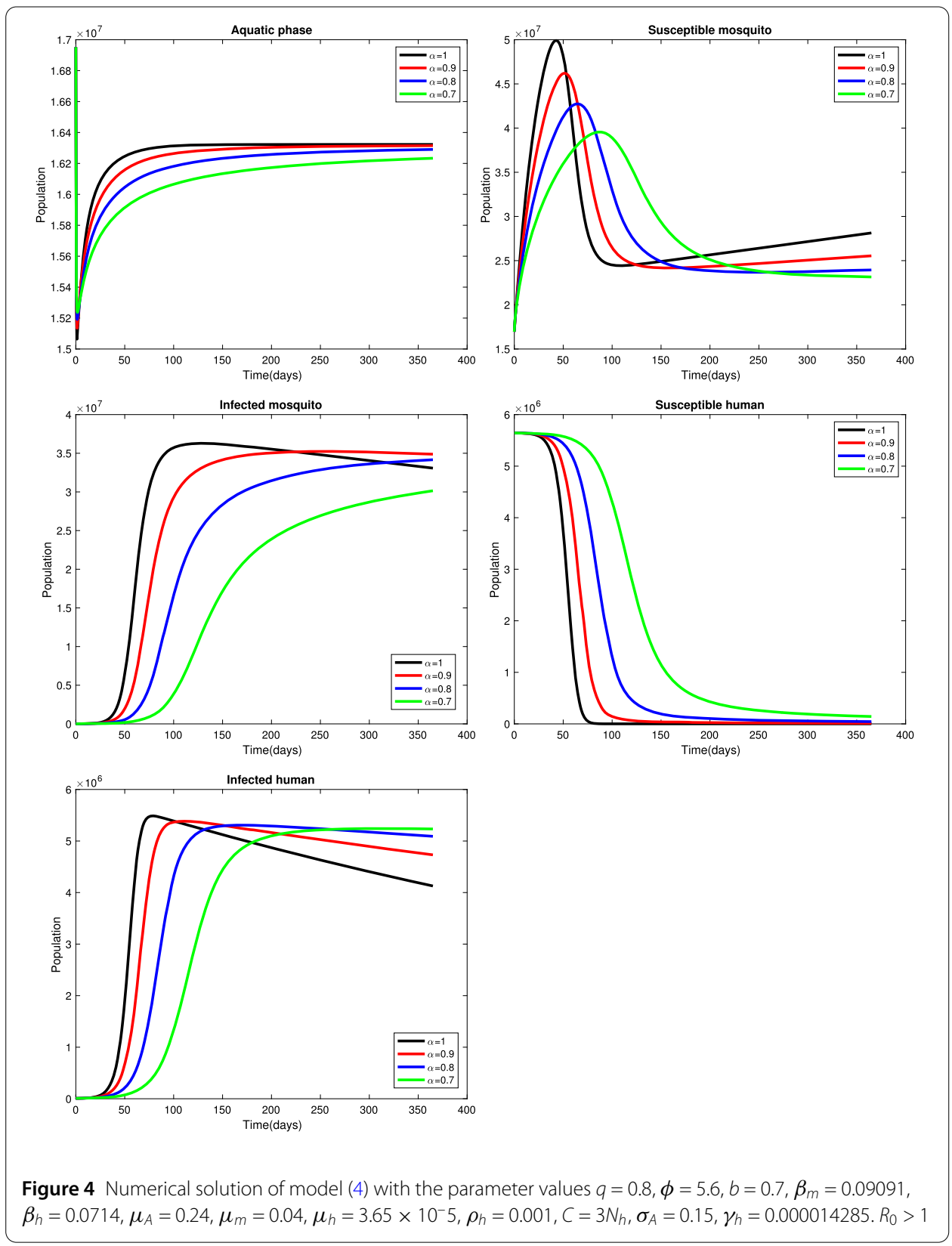

have an increase of the transmission rate of the disease. This tells us that, in the fractional sense, the sensitivity of the biting rate parameter $b$ of the system depends on the $\alpha$ value.

The parameters $\mu_{m}$ and $\gamma_{h}$ have a negative sensitivity index, the most negative being the mortality rate of the mosquito, $\mu_{m}$, with $\Upsilon_{\mu_{m}}^{R_{0}}=-1.0097$. This tells us that if we increase the parameter value of $\mu_{m}$ by $10 \%$, then the basic reproduction number $R_{0}$ will be decreasing by approximately $10 \%$. This agreed with the numerical simulation obtained in Fig. 5 .

\section{Conclusions}

In the present paper, a fractional order dengue epidemic model is studied. The local stability of the equilibrium points has been determined theoretically and verified numerically. The numerical experiment revealed that, for the fractional order model, the speed of con- 

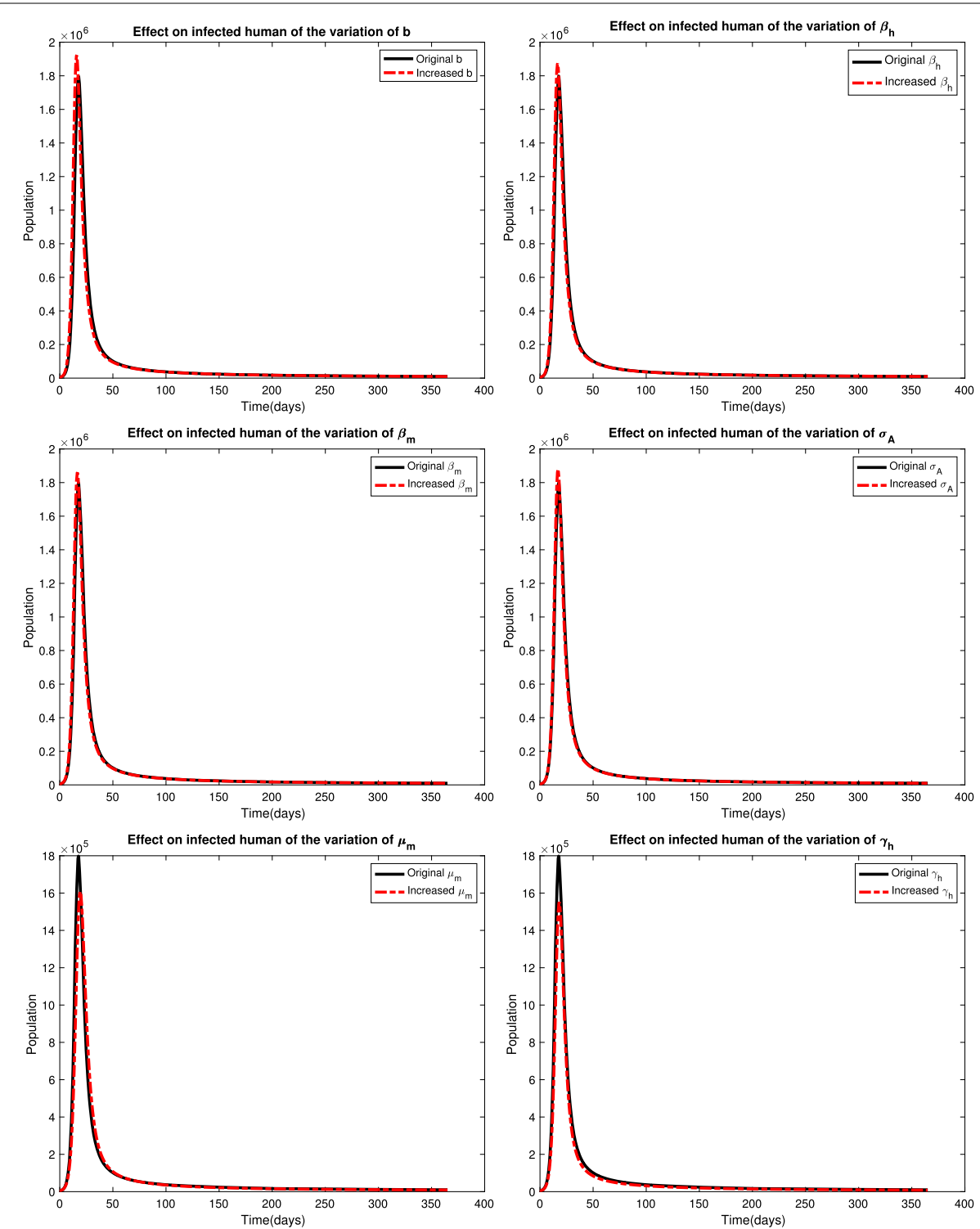

Figure 5 Infected human population with the initial parameter value (solid line) and with an increase of 10\% of a specific parameter (dashed line)

vergence of the solution is slower than in the classical integer model. From the epidemiological point of view, this result is crucial, because it affects the time needed to eliminate the disease.

Also, we have performed a sensitivity analysis in order to determine the relative importance of the model parameters in the transmission of dengue. Such information allows us to determine the effective control and prevention measures, in monitoring the spread of the disease.

We believed that this work can provide an important tool to the public health practitioners in dealing with the increase of dengue cases in the real-life situation, especially in Malaysia. 


\section{Acknowledgements}

The authors would like to thank the editor and reviewers for the constructive comments and useful suggestions, which improved the manuscript.

\section{Funding}

The authors acknowledge financial support by the Ministry of Education Malaysia and Universiti Teknologi MARA and also partial financial support by the Universiti Putra Malaysia providing Putra Grant GP-IPS/2018/9625000.

\section{Availability of data and materials}

Not applicable.

\section{Competing interests}

The authors declare that they have no competing interests.

\section{Authors' contributions}

All authors contributed equally to the manuscript and read and approved the final manuscript.

\section{Author details}

'Department of Mathematics, Faculty of Science, Universiti Putra Malaysia, Selangor, Malaysia. ${ }^{2}$ Institute for Mathematical Research (INSPEM), Universiti Putra Malaysia, Selangor, Malaysia.

\section{Publisher's Note}

Springer Nature remains neutral with regard to jurisdictional claims in published maps and institutional affiliations.

Received: 5 September 2018 Accepted: 20 January 2019 Published online: 28 January 2019

\section{References}

1. World Health Organisation (WHO). Denque. http://www.whho.int/denguecontrol/disease/en. Accessed 10 December 2017

2. Gubler, D.J.: Cities spawn epidemic dengue viruses. Nat. Med. 10, 129-130 (2004)

3. Ahmad, R., Suzilah, I., Wan Nadjah, W.M.A., Topek, O., Mustafakamal, I., Lee, H.L.: Factors determining dengue outbreak in Malaysia. PLoS ONE 13(2), e0193326 (2018)

4. Kermack, W.O., McKendrick, A.G.: A contribution to the mathematical theory of epidemics. Proc. R. Soc. A, Math. Phys. Eng. Sci. 115(772), 700-721 (1927)

5. Bailey, N.T.J.: The Mathematical Theory of Infectious Diseases and Its Applications. Griffin, London (1975)

6. Esteva, L., Vargas, C.: Analysis of dengue transmission model. Math. Biosci. 15(2), 131-151 (1998)

7. Esteva, L., Yang, H.M.: Assessing the effects of temperature and dengue virus load on dengue transmission. J. Biol. Syst. 23(4), 527-554 (2015)

8. Pinho, S.T.R., Ferreira, C.P., Esteva, L., Barreto, F.R., Morato, E., Silva, V.C., Teixeira, M.G.L.: Modelling the dynamics of dengue real epidemics. Philos. Trans. R. Soc. 368, 5679-5693 (2010)

9. Yang, H.M., Ferreira, C.P.: Assessing the effects of vector control on denque transmission. Appl. Math. Comput. 198, 401-413 (2008)

10. Yang, C.X., Nie, L.F.: The effect of vector control strategy against dengue transmission between mosquitoes and human. Electron. J. Qual. Theory Differ. Equ. 2017, 12 (2017)

11. Carvalho dos Santos, J.P., Cardoso, L.C., Monteiro, E., Lemes, N.H.T.: A fractional-order epidemic model for bovine babesiosis disease and tick populations. Abstr. Appl. Anal. 2015, Article ID 729894 (2015)

12. Area, I., Batarfi, H., Losada, J., Nieto, J.J., Shammakh, W., Torres, A.: On a fractional order Ebola epidemic model. Adv. Differ. Equ. 2015, 278 (2015)

13. Area, I., Losada, J., Ndairou, F., Nieto, J.J., Tcheutia, D.D.: Mathematical modeling of 2014 Ebola outbreak. Math. Methods Appl. Sci. 40, 6114-6122 (2017)

14. Al-Sulami, H., El-Shahed, M., Nieto, J.J., Shammakh, W.: On fractional order dengue epidemic model. Math. Probl. Eng. 2014, Article ID 456537 (2014)

15. Pooseh, S., Rodrigues, H., Torres, D.: Fractional derivatives in dengue epidemics. In: Simos T., Psihoyios G., Tsitouras C., Anastassi Z. (eds.) Numerical Analysis and Applied Mathematics ICNAAM, pp. 739-742. Am. Inst. of Phys., Melville (2011)

16. Diethelm, K:: A fractional calculus based model for the simulation of an outbreak of dengue fever. Nonlinear Dyn. 71(4), 613-619 (2013)

17. Sardar, T., Rana, S., Chattopadhyay, J.: A mathematical model of dengue transmission with memory. Commun. Nonlinear Sci. Numer. Simul. 22, 511-525 (2014)

18. Sardar, T., Rana, S., Bhattacharya, S., Al-Khaled, K., Chattopadhyay, J.: A generic model for a single strain mosquito-transmitted disease with memory on the host and the vector. Math. Biosci. 263, 18-36 (2015)

19. Kilbas, A.A., Srivastava, H.M., Trujillo, J.J: Theory and Applications of Fractional Differential Equations. North-Holland Mathematics Studies. Elsevier, Amsterdam (2006)

20. Hamdan, N.I., Kilicman, A.: A fractional order SIR epidemic model for dengue transmission. Chaos Solitons Fractals 114(2018), 55-62 (2018)

21. Syafruddin, S., Noorani, M.S.M.: SEIR model for transmission of dengue fever in Selangor Malaysia. Int. J. Mod. Phys. Conf. Ser. 9, 380-389 (2012)

22. Ministry of Health Malaysia. Health Facts 2012. http://www.moh.gov.my. Accessed 10 February 2018

23. Ang, K.C., Li, Z: Modelling the spread of dengue in Singapore. In: Conference Proceedings for the International Congress on Modeling and Simulation, Hamilton, New Zealand, 1999, vol. 2, pp. 555-560 (2002) 
24. Garba, S.M., Gumel, A.B., Abu Bakar, M.R.: Backward bifurcations in dengue transmission dynamics. Math. Biosci. 215(1), 11-25 (2008)

25. Hethcote, H.W.: The mathematics of infectious disease. SIAM Rev. 42(2), 599-653 (2000)

26. van den Driessche, P., Watmough, J.: Reproduction numbers and sub-threshold endemic equilibria for compartmental models of disease transmission. Math. Biscay 180, 29-48 (2002)

27. Rodrigues, H.S., Teresa, M., Monteiro, T., Torres, D.F.M.: Sensitivity analysis in a dengue epidemiological model. Conference Papers in Mathematics (2013)

28. Chitnis, N., Hyman, J.M., Cushing, J.M.: Determining important parameters in the spread of malaria through the sensitivity analysis of a mathematical model. Bull. Math. Biol. 70(5), 1272-1296 (2008)

29. data.gov.my. MOH Denggue Mortality 2010-2015. http://www.data.gov.my. Accessed 21 March 2018

30. Garrappa, R.: Trapezoidal methods for fractional differential equations: theoretical and computational aspects. Math Comput. Simul. 11, 96-112 (2015)

\section{Submit your manuscript to a SpringerOpen ${ }^{\circ}$} journal and benefit from:

- Convenient online submission

Rigorous peer review

Open access: articles freely available online

High visibility within the field

- Retaining the copyright to your article

Submit your next manuscript at $\gg$ springeropen.com 